\title{
Neutron Skin and Giant Resonances in Nuclei
}

\author{
Vadim Rodin \\ Institute for Theoretical Physics, University of Tübingen \\ Auf der Morgenstelle 14, D-72076 Tübingen, Germany
}

November 1, 2018

\begin{abstract}
Some aspects, both experimental and theoretical, of extracting the neutron skin $\Delta R$ from properties of isovector giant resonances are discussed. Existing proposals are critically reviewed. The method relying on the energy difference between the GTR and IAS is shown to lack sensitivity to $\Delta R$. A simple explanation of the linear relation between the symmetry energy and the neutron skin is also presented.
\end{abstract}

\section{Introduction}

Accurate experimental data on the neutron skin in neutron rich nuclei would allow to further constrain model parameters involved in the calculations of the nuclear symmetry energy [1]. The latter plays a central role in a variety of nuclear phenomena. The value $a_{4} \approx 30 \mathrm{MeV}$ of the nuclear symmetry energy $S\left(\rho_{0}\right)=a_{4}+\frac{p_{0}}{\rho_{0}^{2}}\left(\rho-\rho_{0}\right)+\ldots$ at nuclear saturation density $\rho_{0} \approx 0.17 \mathrm{fm}^{-3}$ seems reasonably well established. On the other hand, the density dependence of the symmetry energy can vary substantially with the many-body approximations employed.

Several authors have pointed out [2, 3] a strong correlation between the neutron skin, $\Delta R=\sqrt{\left\langle r^{2}\right\rangle_{n}}-$ $\sqrt{\left\langle r^{2}\right\rangle_{p}}=R_{n}-R_{p}$, and the symmetry energy of neutron matter near saturation density. In the framework of a mean field approach Furnstahl [3] demonstrated that in heavy nuclei there exists an almost linear empirical correlation between theoretical predictions in terms of various mean field approaches to $S(\rho)$ (i.e., a bulk property) and the neutron skin, $\Delta R$ (a property of finite nuclei).

This observation has contributed to a renewed interest in an accurate determination of the neutron skin in neutron rich nuclei. Besides, a precise value of the neutron skin is required as an input in several processes of physical interest, e.g. the analysis of energy shifts in deeply bound pionic atoms [4, and in the analysis of atomic parity violation experiments (weak charge) [5]. It is worth to stress that to experimentally determine the skin in heavy nuclei is extremely challenging as $\Delta R$ is just about few percents of the nuclear radius.

The present contribution is partially based upon the results published previously in [6].

\section{Relationship between the symmetry energy and $\Delta R$}

Brown [2] and Furnstahl [3] have pointed out that within the framework of mean field models there exists an almost linear empirical correlation between theoretical predictions for both $a_{4}$ and its density 
dependence, $p_{0}$, and the neutron skin $\Delta R$ in heavy nuclei. This observation suggests an intriguing relationship between a bulk property of infinite nuclear matter and a surface property of finite systems. Here, following the analysis of [6], this question is addressed from a point of view of the Landau-Migdal approach.

Let us consider a simple mean-field model with the Hamiltonian consisting of the single-particle mean field part $\hat{H}_{0}$ and the residual particle-hole interaction $\hat{H}_{p-h}$ :

$$
\begin{gathered}
\hat{H}=\hat{H}_{0}+\hat{H}_{p-h}, \quad \hat{H}_{p h}=\sum_{a>b}\left(F^{\prime}+G^{\prime} \vec{\sigma}_{a} \vec{\sigma}_{b}\right) \vec{\tau}_{a} \vec{\tau}_{b} \delta\left(\vec{r}_{a}-\vec{r}_{b}\right), \\
\hat{H}_{0}=\sum_{a}\left(T_{a}+U\left(x_{a}\right)\right), \quad U(x)=U_{0}(x)+U_{1}(x)+U_{C}(x), \\
U_{0}(x)=U_{0}(r)+U_{s o}(x) ; \quad U_{1}(x)=\frac{1}{2} S_{\mathrm{pot}}(r) \tau^{(3)} ; \quad U_{C}(x)=\frac{1}{2} U_{C}(r)\left(1-\tau^{(3)}\right) .
\end{gathered}
$$

Here, $U_{0}(x)$ is the phenomenological isoscalar part of the mean field potential $U(x)(x=\{\vec{r}, \vec{\sigma}, \vec{\tau}\})$, $U_{0}(r)$ and $U_{s o}(x)$ are the central and spin-orbit parts, respectively; $F^{\prime}$ and $G^{\prime}$ are the phenomenological Landau-Migdal parameters. The isovector part $U_{1}(x)$ and the Coulomb mean field $U_{C}(x)$ are both calculated consistently in the Hartree approximation, $S_{\text {pot }}(r)$ is the symmetry potential $(r$-dependent symmetry energy in finite nuclei).

The model Hamiltonian $\hat{H}$ (1) preserves the isospin symmetry within the RPA if a selfconsistency relation between the symmetry potential and the Landau-Migdal parameter $F^{\prime}$ is fulfilled:

$$
S_{\text {pot }}(r)=2 F^{\prime} n^{(-)}(r)
$$

where $n^{(-)}(r)=n_{n}(r)-n_{p}(r)$ is the neutron excess density. Thus, in this model the depth of the symmetry potential is controlled by the Landau-Migdal parameter $F^{\prime}$ (analogous role plays the parameter $g_{\rho}^{2}$ in relativistic mean field models). $S_{\text {pot }}(r)$ is obtained from Eq.(4) by an iterative procedure; the resulting dependence of $\Delta R$ on the dimensionless parameter $f^{\prime}=F^{\prime} /\left(300 \mathrm{MeV} \mathrm{fm}^{3}\right)$ shown in fig. 1 indeed illustrates that $\Delta R$ depends almost linearly on $f^{\prime}$. Then with the use of the Migdal relation $a_{4}=\frac{\epsilon_{F}}{3}\left(1+2 f^{\prime}\right)$ [7] relating the symmetry energy and $f^{\prime}$, a similar, almost linear, correlation between $a_{4}$ and $\Delta R$ is obtained.

To get more insight in the role of $f^{\prime}$ we consider small variations $\delta F^{\prime}$. Neglecting the variation of $n^{(-)}(r)$ with respect to $\delta F^{\prime}$, the corresponding linear variation of the symmetry potential is $\delta S_{p o t}(r)=2 \delta F^{\prime} n^{(-)}(r)$. Then in the first order perturbation theory, such a variation of $S_{p o t}$ causes the following variation of the ground-state wave function $|\delta 0\rangle=\delta F^{\prime} \sum_{s} \frac{\left\langle s\left|\hat{N}^{(-)}\right| 0\right\rangle}{E_{0}-E_{s}}|s\rangle$, with " $s$ " labeling the eigenstates of the nuclear Hamiltonian and a single-particle operator $\hat{N}^{(-)}$defined as $\hat{N}^{(-)}=\sum_{a} n^{(-)}\left(r_{a}\right) \tau_{a}^{(3)}$. Consequently, the variation of the expectation value $\left\langle 0\left|\hat{V}^{(-)}\right| 0\right\rangle=N R_{n}^{2}-Z R_{p}^{2}$ of another single-particle operator $\hat{V}^{(-)}=\sum_{a} r_{a}^{2} \tau_{a}^{(3)}$ can be written as

$$
R_{p} \delta(\Delta R)=\delta F^{\prime} \cdot \frac{2}{A} \sum_{s} \frac{\operatorname{Re}\left\langle 0\left|\hat{N}^{(-)}\right| s\right\rangle\left\langle s\left|\hat{V}^{(-)}\right| 0\right\rangle}{E_{0}-E_{s}}
$$

In practice the sum in Eq. (5) is exhausted mainly by the isovector monopole resonance (IMR) which high excitation energy (about $24 \mathrm{MeV}$ in ${ }^{208} \mathrm{~Pb}$ ) justifies the perturbative consideration. Eq. (5) is able to reproduce directly calculated $\delta(\Delta R)$ shown in Fig. 11 with the accuracy of about $10 \%$. As a result, a simple microscopic interpretation of the linear correlation between $\Delta R$ and Landau parameter $F^{\prime}$ is obtained. 


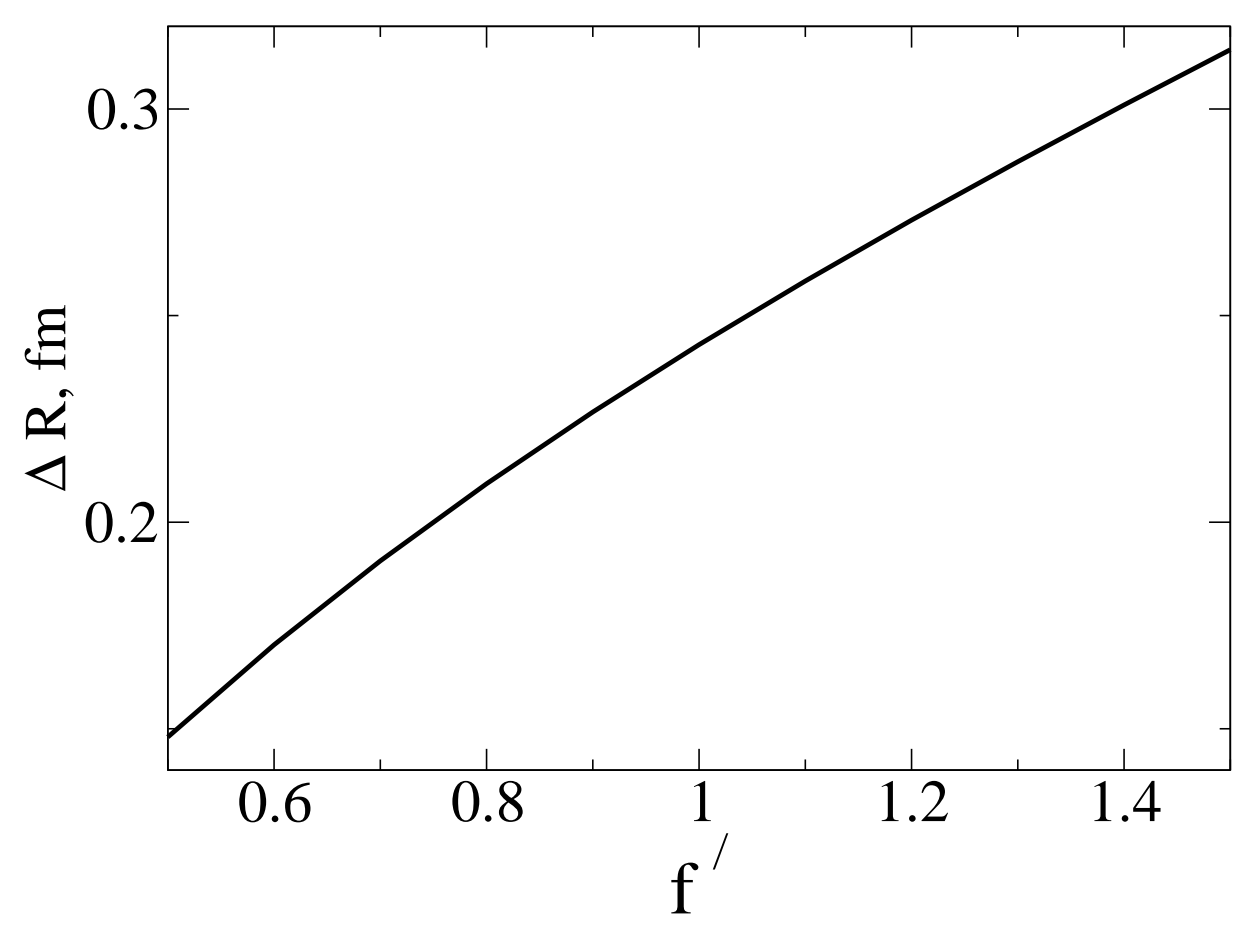

Figure 1: Neutron skin in ${ }^{208} \mathrm{~Pb}$ versus the Landau-Migdal parameter $f^{\prime}$.

\section{Extracting neutron skin from properties of isovector giant resonances}

Parity violating electron scattering off nuclei is probably the least model dependent approach to probe the neutron distribution [8]. The weak electron-nucleus potential is $\tilde{V}(r)=V(r)+\gamma_{5} A(r)$, where the axial potential $A(r)=\frac{G_{F}}{2^{3 / 2}} \rho_{W}(r)$. The weak charge is mainly determined by neutrons $\rho_{W}(r)=$ $\left(1-4 \sin ^{2} \theta_{W}\right) \rho_{p}(r)-\rho_{n}(r)$, with $\sin ^{2} \theta_{W} \approx 0.23$. In a scattering experiment using polarized electrons one can determine the cross section asymmetry [8] which comes from the interference between the $A$ and $V$ contributions. Using the measured neutron form factor at small finite value of $Q^{2}$ and the existing information on the charge distribution one can uniquely extract the neutron skin. Some slight model dependence comes from the need to assume a certain radial dependence for the neutron density, to extract $R_{n}$ from a finite $Q^{2}$ form factor.

However, the best claimed accuracy of the experimental determination of neutron radii would be on the level of $1 \%$, that translates to relatively large uncertainty of $20-30 \%$ in the neutron skin. On such accuracy level, some indirect experimental probes of $\Delta R$ still can be competitive.

A variety of experimental approaches have been employed to obtain indirect information on $\Delta R$. To some extent all the analysis contain a certain model dependence, which in many cases is difficult to estimate quantitatively. For choosing an indirect probe it is very important to address the question how sensitive is the proposed physical quantity with respect to a variation of $\Delta R$ in a single nucleus. The higher is the sensitivity, the better is the choice of the correlation for the indirect deducing $\Delta R$ from the measured values.

It is not intended here to give a comprehensive review of the existing methods. In particular, the results from the analysis of the antiprotonic atoms, elastic proton and neutron scattering reactions, and the pygmy dipole resonance are completely left out. Here, special emphasis will be put on proposals to provide accurate information on the neutron skin from properties of isovector giant resonances. 


\subsection{Spin-dipole Giant Resonance}

In [9] it has been proposed to utilize the excitation probability of the spin-dipole resonance in charge exchange reactions for determining the neutron skin. The method has been applied to obtain information on the variation of the neutron skin in the Sn isotopes [9]. For the relevant operator, $\sum_{a} \tau_{a}^{ \pm}\left[\vec{\sigma}_{a} \otimes\right.$ $\left.\vec{r}_{a}\right]_{J M},(J=0,1,2)$ the summed $\Delta L=1$ strength is

$$
S^{(-)}-S^{(+)}=C\left(N R_{n}^{2}-Z R_{p}^{2}\right)
$$

Here $S^{(-)}$and $S^{(+)}$are the spin-dipole total strengths in $\beta^{(-)}$and $\beta^{(+)}$channels, respectively; $C$ is the factor depending on the normalization of the spin-dipole operator (in the definition of Ref. [2] $C=1 / 4 \pi$, we use here $C=1$ ). Because $S^{(+)}$could not be measured experimentally, the model-dependent energyweighted sum rule was invoked in the analysis of [9] to eliminate $S^{(+)}$. However, the used analytical representation for the sum rule was oversimplified and led in some cases, e.g. for ${ }^{208} \mathrm{~Pb}$, to absurdly negative $S^{(+)}$. In [10] another way was proposed, namely, to use for the analysis the ratio $S^{(+)} / S^{(-)}$ calculated within the pn-RPA. The parameterization of the RPA calculation results for tin isotopes in the form

$$
S^{(+)} / S^{(-)}=0.388-0.012(N-Z)
$$

was used later in [11] to reanalyze the experimental data and led to a marked change in the extracted $\Delta R$ 's.

Let us now assess the experimental accuracy for $S^{(-)}$needed to determine the neutron skin to a given accuracy. Putting $S^{(+)}=0$ (that seems to be a very good approximation for ${ }^{208} \mathrm{~Pb}$ ) and one has

$$
S^{(-)}=(N-Z) R_{p}^{2}+2 N R_{p} \Delta R .
$$

The ratio of the second term on the rhs to the first one in case of ${ }^{208} \mathrm{~Pb}$ is

$$
2 N \Delta R /\left((N-Z) R_{p}\right) \approx 5.7 \Delta R / R_{p}
$$

Therefore, for $R_{p}=5.5 \mathrm{fm}$ and $\Delta R=0.2 \mathrm{fm}$ the second term is only $25 \%$ of the first one and one needs $5 \%$ accuracy in $S^{(-)}$to determine $\Delta R$ with $20 \%$ accuracy. Because the SD strength is spread out and probably has a considerable strength at low-energy, the results for the $\Delta R$ can be only considered as qualitative with a relatively large uncertainty (up to 30-50\%).

\subsection{Isobaric analogue state}

The dominant contribution to the energy weighted sum rule (EWSR) for Fermi excitations by the operator $T^{(-)}=\sum_{a} \tau_{a}^{-}$comes from the Coulomb mean field

$$
(E W S R)_{F}=\int U_{C}(r) n^{(-)}(r) d^{3} r
$$

The Coulomb mean field $U_{C}(r)$ resembles very much that of the uniformly charged sphere, being inside a nucleus a quadratic function: $U_{C}(r)=\frac{Z e^{2}}{2 R_{c}}\left(3-\left(r / R_{c}\right)^{2}\right), r \leq R_{c}$. It turns out that if one extends such a quadratic dependence also to the outer region $r>R_{c}$ (instead of proportionality to $R_{c} / r$ ), it gives numerically just a very small deviation in $(E W S R)_{F}$ (less than $0.5 \%$, due to the fact, that both the difference and its first derivative go to zero at $r=R_{c}$ and $n^{(-)}(r)$ is exponentially decreasing for $r>R_{c}$ ). Using such an approximation, one gets:

$$
(E W S R)_{F} \approx(N-Z) \Delta_{C}\left(1-\frac{S^{(-)}}{3(N-Z) R_{c}^{2}}\right)
$$


with $\Delta_{C}=\frac{3 Z e^{2}}{2 R_{c}}$, and $S^{(-)}$given in Eq.(7).

Since the IAS exhausts almost $100 \%$ of the NEWSR and EWSR, one may hope to extract $S^{(-)}$from the IAS energy. However, the term depending on $S^{(-)}$contributes only about $20 \%$ to $(E W S R)_{F}$, and as a result, the part of $S^{(-)}$depending on $\Delta R$ contributes only about $4 \%$ to $(E W S R)_{F}$ (in $\left.{ }^{208} \mathrm{~Pb}\right)$. ¿From the experimental side, the IAS energy can be determined with unprecendently high accuracy, better than $0.1 \%$. Also, from the experimentally known charge density distribution the Coulomb mean field $U_{C}(r)$ can be calculated rather accurately, and hence one can determine the small difference between Eqs.(9) and (8). But at the level of $1 \%$ accuracy several theoretical effects discarded in Eq.(8) come into play that makes such an accurate description of the IAS energy very difficult (the Nolen-Schiffer anomaly).

Also in [12] it was stated that the Coulomb displacement energies (CDE) are sensitive to $\Delta R$. A gross estimate $\Delta R=0.80(5)(N-Z) / A$ fm was obtained from a four-parameter fit of the experimental $R_{p}$ and observed mirror CDE's. The authors claimed $127 \mathrm{keV}$ to be the rms error of the fit, but they assumed the nuclear wave functions calculated within the Nuclear Shell Model to be isospin pure. Thus, the important effect of the Coulomb mixing of the IAS and the IMR was not taken into account, which is known to decrease the IAS energy by a few percents. Therefore, the Nolen-Schiffer anomaly does not seem to have been resolved yet.

\subsection{Is the energy spacing between GTR and IAS a good candidate for determining the neutron skin in isotopic chains?}

In a recent paper [13] a proposal has been put forward to use the isotopic dependence of the energy spacing, $\Delta E$, between the Gamow-Teller resonance (GTR) and the IAS as a tool for determining the evolution of the neutron skin in nuclei along an isotopic chain. Here, we would like to present some physical arguments which question the physical relevance of this method.

The authors of [13] have used the fact that both functions, $\Delta R$ and $\Delta E$, are monotonic functions (increasing and decreasing, respectively) of the neutron excess $(N-Z)$ to state that "isotopic dependence of the energy spacings between the GTR and IAS provides direct information on the evolution of neutron skin-thickness along the Sn isotopic chain". Arguing in such a way one can find a correlation between any two monotonic functions of a single physical parameter and plot them as a function of one another like is done in Fig. 2 of [13] 1. However, it does not imply automatically a real physical correlation between the functions which are determined also by many other model parameters which are kept fixed while performing calculations (the calculations in [13] have been performed within the relativistic mean field (RMF) and relativistic QRPA (RQRPA) approaches).

Again, the relevant question to be addressed is how sensitive is one physical quantity with respect to a variation of another in a single nucleus? In other words, one has to evaluate what variation of $\Delta E$ is produced by varying $\Delta R$ in a single nucleus. Imaging an extreme situation (which is actually not far from reality) that $\Delta E$ were not sensitive to $\Delta R$ at all, one would get by varying $\Delta R$ a family of different calculated dependences (like shown in the upper panel of Fig. 2 of [13]) which would give no clue about the real dependence seen in nature.

Thus, it is quite important to understand the physical reasons which cause the energy splitting between the GTR and the IAS. It is well-known that if the nuclear Hamiltonian possessed Wigner $\mathrm{SU}(4)$ symmetry then the GTR and the IAS would be degenerate, $\Delta E=0$. In such a case any variation of $\Delta R$, not violating the symmetry, would not affect $\Delta E$ at all. However, it is also known that the spin-isospin $\mathrm{SU}(4)$ symmetry is broken in nuclei. Hence, $\Delta E$ is determined by those terms in

\footnotetext{
${ }^{1}$ note that, to avoid confusion in comparing the measured and calculated dependences, the authors should have plotted the experimental points in the upper panel as the function of the measured $\Delta R$ rather than calculated $\Delta R$ and should have added the horizontal error bars to them reflecting the experimental uncertainty in $\Delta R$ shown in the lower panel.
} 
the nuclear Hamiltonian which violate the symmetry. Their qualitative and semi-quantitative estimates in terms of the energy weighted sum rules for the Gamow-Teller $\left(E W S R_{G T}\right)$ and the Fermi $\left(E W S R_{F}\right)$ excitations have been already known for more than 20 years (see, e.g., [14]). The analysis of these authors as well as a quantitative analysis performed recently in [15] has shown that there are three basic sources in the Hamiltonian which violate SU(4) symmetry and contribute to the difference of the sum rules: spin-orbit mean field and both particle-particle and particle-hole residual charge-exchange interactions. One sees that none of the sources explicitly refers to the symmetry potential, to which $\Delta R$ is especially sensitive.

An estimate of $\Delta E$ as $\Delta E=\frac{E W S R_{G T}-E W S R_{F}}{N-Z}$ can be calculated according to [15] in the Sn isotopes. From the sources violating SU(4) symmetry, spin-orbit mean field represents the major one and contributes about $5 \mathrm{MeV}$ to the splitting. The contribution of the particle-hole interaction is negative and about $1-2 \mathrm{MeV}$ in the absolute value. The contribution of the particle-particle interaction is rather difficult to evaluate (due to uncertainty in the strength of the spin-dependent particle-particle interaction) but it seems to be of minor importance (very probably no more than $0.5 \mathrm{MeV}$, especially for large $(N-Z))$ and can safely be neglected.

Now let us turn to the discussion of the sensitivity of the contributions to the variation of $\Delta R$. We could reproduce the corresponding analytical expressions from [15] explicitly, but it is enough for our purpose just to mention that the dominating contribution to $\Delta E$ from the spin-orbit mean field is given by its expectation value in the ground state and is determined basically only by the unfilled spin-orbit doublets. This expectation value is completely insensitive to the variation of $\Delta R$.

Within the Landau-Migdal approach described above, the particle-hole contribution

$$
\Delta E_{p h}=\frac{2\left(G^{\prime}-F^{\prime}\right)}{N-Z} \int\left(n^{(-)}(r)\right)^{2} d^{3} r
$$

is given by the product of the volume integral of the neutron excess density squared and the difference of the p-h strengths $G^{\prime}$ and $F^{\prime}\left[15\right.$. In the $S U(4)$-symmetric limit one has $G^{\prime}=F^{\prime}$ and $\Delta E_{p h}=0$ explicitly. Still, in this limit one has a freedom to choose different $F^{\prime}$ that produces a variation in $\Delta R$, similar to shown in fig 1 . Therefore, as already mentioned, one can get no clue about the actual $\Delta R$ from $\Delta E=0$ in the $S U(4)$-symmetric limit.

In a realistic situation $G^{\prime} \neq F^{\prime}\left(f^{\prime}=1.0\right.$ and $g^{\prime}=0.8$ were taken in [15]), but $\Delta E_{p h}$ depends only on the difference $G^{\prime}-F^{\prime}$. One usually fixes $G^{\prime}$ in order to reproduce the GTR energy in some nuclei (the authors of [13] have followed this way, too) and possible information from $\Delta E$ about the absolute value of $F^{\prime}$ is lost. Furthermore, one can a priori think that a degree of violation of the SU(4) symmetry should be a sort of a fundamental property of the residual interaction. Therefore, the difference $G^{\prime}-F^{\prime}$ should stay more stable in different models as compared to some possible variation of $F^{\prime}$ producing different $\Delta R$.

Considering value of $G^{\prime}-F^{\prime}$ fixed, one can employ a simple model varying only $\rho_{n}(r)$ to see how a change of $\Delta R$ affects $\Delta E_{p h}$ via variation of the neutron excess density $n^{(-)}(r)$. A small variation of $\rho_{n}(r)$ can be approximately represented as $\delta \rho_{n}(r)=-\frac{\delta R_{n}}{R_{n}}\left(3 \rho_{n}(r)+R \frac{d \rho_{n}(r)}{d r}\right)$, where $\delta R_{n}$ is a change of the rms neutron radius $R_{n}, R$ is the nuclear radius (with $R_{n}^{2} \approx 0.6 R^{2}$ ). Assuming the proton and neutron densities be constant inside a nucleus, the final estimate is $\frac{\delta \Delta E_{p h}}{\Delta E_{p h}}=-3 \frac{\delta R_{n}}{R_{n}}\left(1+\frac{N+Z-2 \gamma N}{N-Z}\right)$, where $\gamma=n^{(-)}(R) / n^{(-)}(0)$

Thus, in Sn isotopes with the experimental charge radii about $R_{p}=4.6 \mathrm{fm}$ a rather significant variation of $\Delta R$ about $0.1 \mathrm{fm}$, that is of the order of magnitude of $\delta R$, would cause $\frac{\delta \Delta E_{p h}}{\Delta E_{p h}}=0.3$ and $\frac{\delta \Delta E_{p h}}{\Delta E_{p h}}=0.15$ for ${ }^{112} \mathrm{Sn}$ and ${ }^{132} \mathrm{Sn}$, respectively $(\gamma=0.5)$, that corresponds to the absolute change about $0.3 \mathrm{MeV}$ in $\Delta E$, to be compared with the experimental uncertainties in $\Delta E$ of the same order. It 
is clear that to draw any conclusion about $\Delta R$ from the measured $\Delta E$ would be premature. Even if the experimental errors in $\Delta E$ were exactly zero, the accuracy of the theoretical model itself would be hardly believed to be of the necessary level. For instance, apart from the obvious uncertainties in the isotopic dependence of the spin-orbit potential, the GTR does not exhaust $100 \%$ of the corresponding sum rules and the shell-structure effects such as configurational and isospin splitting of the GTR can have some effect on the calculated GTR energy.

It is also noteworthy that, in spite of the claimed self-consistency of the calculations, the slope of the calculated isotopic dependence of the IAS energy is about 3 times larger than the experimental one (see inset in Fig. 1 of [13]). Note, that the isospin self-consistent continuum-QRPA calculations of [15] were able to nicely reproduce the slope (while overall underestimated the IAS energy by about $0.5 \mathrm{MeV}$, the well-known Nolen-Schiffer anomaly).

To conclude, we believe that the suggested in [13] method to deduce the neutron skin from the energy spacing between GTR and IAS is rather questionable in its origin and does not fairly provide "direct information on the evolution of neutron skin-thickness".

\section{Some implications of $\Delta R$}

In several processes of physical interest knowledge of $\Delta R$ plays a crucial role and in fact a more accurate value could lead to more stringent tests:

(i) The pion polarization operator [4] (the s-wave optical potential) in a heavy nucleus $\Pi\left(\omega, \rho_{p}, \rho_{n}\right)=$ $-T^{+}(\omega) \rho-T^{-}(\omega)\left(\rho_{n}-\rho_{p}\right)$ has mainly an isovector character $\left(T^{+}\left(m_{\pi}\right) \sim 0\right)$. Parameterizing the densities by Fermi shapes for the case of ${ }^{208} \mathrm{~Pb}$ the main nuclear model dependence in the analysis comes from the uncertainty in the value of $\Delta R$ multiplying $T^{-}$.

(ii) The parity violation in atoms is dominated by $Z$-boson exchange between the electrons and the neutrons [5]. Taking the proton distribution as a reference there is a small so-called neutron skin (ns) correction to the parity non-conserving amplitude, $\delta E_{\mathrm{pnc}}^{\mathrm{ns}}$, for, say, a $6 s_{1 / 2} \rightarrow 7 s_{1 / 2}$ transition, which is related to $\Delta R$ as (independent of the electronic structure)

$$
\frac{\delta E_{\mathrm{pnc}}^{\mathrm{ns}}}{E_{\mathrm{pnc}}}=-\frac{3}{7}(\alpha Z)^{2} \frac{\Delta R}{R_{p}} .
$$

In ${ }^{133} \mathrm{Cs}$ it amounts to a $\delta E / E \approx-(0.1-0.4) \%$ depending on whether the non-relativistic or relativistic estimates for $\Delta R$ are used [5]. The corresponding uncertainty in the weak charge $Q_{W}$ is $-(0.2-0.8) \sigma$.

(iii) The pressure in a neutron star matter can be expressed as in terms of symmetry energy and its density dependence

$$
P(\rho, x)=\rho^{2} \frac{\partial E(\rho, x)}{\partial \rho}=\rho^{2}\left[E^{\prime}(\rho, 1 / 2)+S^{\prime}(\rho)(1-2 x)^{2}+\ldots\right] .
$$

By using beta equilibrium in a neutron star, $\mu_{e}=\mu_{n}-\mu_{p}=-\frac{\partial E(\rho, x)}{\partial x}$, and the result for the electron chemical potential, $\mu_{e}=3 / 4 \hbar c x\left(3 \pi^{2} \rho x\right)^{1 / 3}$, one finds the proton fraction at saturation density, $\rho_{0}$, to be quite small, $x_{0} \sim 0.04$. Hence, the pressure at saturation density can be approximated as

$$
P\left(\rho_{0}\right)=\rho_{s}\left(1-2 x_{0}\right)\left(\rho_{0} S^{\prime}\left(\rho_{0}\right)\left(1-2 x_{0}\right)+S\left(\rho_{0}\right) x_{0}\right) \sim \rho_{0}^{2} S^{\prime}\left(\rho_{0}\right) .
$$

At higher densities the proton fraction increases; this increase is more rapid in case of larger $p_{0}$ [1]. While for the pressure at higher densities contributions from other nuclear quantities like compressibility will play a role in it was argued that that there is a correlation of the neutron star radius and the pressure which does not depend on the EoS at the highest densities. Numerically the correlation can be expressed in the form of a power law, $R_{M} \sim C(\rho, M)\left(\frac{P(\rho)}{\mathrm{MeVfm}^{-3}}\right)^{0.25} \mathrm{~km}$, where $C\left(\rho=1.5 \rho_{s}, M=1.4 M_{\text {solar }}\right) \sim 7$. This shows that a determination of a neutron star radius would provide some constraint on the symmetry properties of nuclear matter. 


\section{Conclusion}

In this contribution we discuss some aspects of extracting the neutron skin from properties of isovector giant resonances and critically review existing proposals. The theoretical method relying on the energy difference between the GTR and IAS is shown to lack sensitivity to $\Delta R$. It is also shown that the phenomenological, almost linear, relationship between the symmetry energy and the neutron skin in finite nuclei, observed in mean field calculations, can be understood in terms the Landau-Migdal approach.

\section{Acknowledgments}

The work is supported in part by the Deutsche Forschungsgemeinschaft (grant FA67/28-2) and by the EU ILIAS project (contract RII3-CT-2004-506222). The author would like to thank Profs. L. Dieperink and M. Urin for useful discussions.

\section{References}

[1] C.J. Horowitz and J. Piekarewicz, Phys. Rev. Lett. 86 (2001) 5647 ; Phys. Rev. C 66 (2002) 055803 .

[2] B.A. Brown, Phys. Rev. Lett. 85 (2000) 5296 .

[3] R. J. Furnstahl, Nucl. Phys. A 706 (2002) 85.

[4] E.E. Kolomeitsev, N. Kaiser and W. Weise, Phys. Rev. Lett. 90 (2003) 092501.

[5] S. J. Pollock and M. C. Welliver, Phys. Lett. B 464 (1998) 177 .

[6] A.E.L. Dieperink, Y. Dewulf, D. Van Neck, M. Waroquier, V. Rodin, Phys. Rev. C 68 (2003) 064307 .

[7] A.B. Migdal, Theory of finite Fermi-systems and properties of atomic nuclei (Moscow, Nauka, 1983) (in Russian).

[8] C. J. Horowitz, S. J. Pollock, P. A. Souder and R. Michaels, Phys. Rev. C 63 (2001) 025501 ; http://hallaweb.jlab.org/parity/prex.

[9] A. Krasznohorkay et al., Phys. Rev. Lett. 82 (1999) 3216 .

[10] V. Rodin, M. Urin, KVI annual report (2000).

[11] M. Csatlós et al., Acta Phys. Polonica B33 (2002) 331 .

[12] J. Duflo and A. P. Zuker, Phys. Rev. C 66 (2002) 051304.

[13] D. Vretenar, N. Paar, T. Nikšić, P. Ring, Phys. Rev. Lett. 91 (2003) 262502.

[14] Yu.V. Gaponov, Yu.S. Lyutostansky, V.G. Aleksankin, JETP Lett. 34 (1981) 386 ; T. Suzuki, Phys. Lett. B 104 (1981) 92 ; K. Nakayama, A. Pio Galeao, F. Krmpotic, Phys. Lett. B 114 (1982) 217.

[15] V.A. Rodin and M.H. Urin, Phys. At. Nuclei 66 (2003) 2128 , nucl-th/0201065. 